\title{
RAF inhibitors prime wild-type RAF to activate the MAPK pathway and enhance growth
}

\author{
Georgia Hatzivassiliou ${ }^{1}$, Kyung Song ${ }^{1}$, Ivana Yen ${ }^{1}$, Barbara J. Brandhuber ${ }^{2}$, Daniel J. Anderson ${ }^{1}$, Ryan Alvarado ${ }^{1}$, \\ Mary J. C. Ludlam¹, David Stokoe ${ }^{1}$, Susan L. Gloor ${ }^{2}$, Guy Vigers², Tony Morales², Ignacio Aliagas ${ }^{1}$, Bonnie Liu', \\ Steve Sideris ${ }^{1}$, Klaus P. Hoeflich ${ }^{1}$, Bijay S. Jaiswal ${ }^{1}$, Somasekar Seshagiri ${ }^{1}$, Hartmut Koeppen ${ }^{1}$, Marcia Belvin ${ }^{1}$, \\ Lori S. Friedman ${ }^{1} \&$ Shiva Malek ${ }^{1}$
}

Activating mutations in KRAS and BRAF are found in more than $30 \%$ of all human tumours and $40 \%$ of melanoma, respectively, thus targeting this pathway could have broad therapeutic effects ${ }^{1}$. Small molecule ATP-competitive RAF kinase inhibitors have potent antitumour effects on mutant $\mathrm{BRAF}(\mathrm{V} 600 \mathrm{E})$ tumours but, in contrast to mitogen-activated protein kinase kinase (MEK) inhibitors, are not potent against RAS mutant tumour models, despite RAF functioning as a key effector downstream of RAS and upstream of $\mathrm{MEK}^{2,3}$. Here we show that ATP-competitive RAF inhibitors have two opposing mechanisms of action depending on the cellular context. In BRAF(V600E) tumours, RAF inhibitors effectively block the mitogen-activated protein kinase (MAPK) signalling pathway and decrease tumour growth. Notably, in KRAS mutant and RAS/RAF wild-type tumours, RAF inhibitors activate the RAF-MEK-ERK pathway in a RAS-dependent manner, thus enhancing tumour growth in some xenograft models. Inhibitor binding activates wild-type RAF isoforms by inducing dimerization, membrane localization and interaction with RAS-GTP. These events occur independently of kinase inhibition and are, instead, linked to direct conformational effects of inhibitors on the RAF kinase domain. On the basis of these findings, we demonstrate that ATP-competitive kinase inhibitors can have opposing functions as inhibitors or activators of signalling pathways, depending on the cellular context. Furthermore, this work provides new insights into the therapeutic use of ATP-competitive RAF inhibitors.

The RAS-RAF-MEK-ERK signalling pathway is an attractive target for therapeutic intervention in oncology, and several selective RAF and MEK small molecule inhibitors are being tested at present in phase I and phase II clinical trials. Although both RAF and MEK (also known as MAP2K) inhibitors have excellent preclinical activity in tumour models with BRAF(V600E) mutations, their potencies in BRAF wild-type (BRAF-WT) and KRAS mutant (KRAS-MT) tumour models unexpectedly diverge. The selective and chemically unrelated RAF inhibitors GDC-0879 (refs 3, 4) and PLX4720 (ref. 5) both show specificity towards BRAF(V600E) tumour lines, unlike the MEK inhibitor PD0325901 that inhibits proliferation of BRAF(V600E), RAS/RAF-WT and KRAS-MT tumour lines (Fig. 1a). Unexpectedly, RAF inhibitors cause an increase in viable cell numbers (marked with an asterisk in Fig. 1a; see also Supplementary Fig. 2) in a subset of BRAF-WT tumour cell lines ( $~ 50 \%$ of tested). Furthermore, although GDC-0879 is able to inhibit growth of BRAF(V600E) tumour xenografts in vivo, it can increase the growth rate of KRAS-MT lung xenografts (Fig. 1b). In addition, histopathological examination of mice treated with GDC-0879 showed hyperkeratosis and acanthosis of the epidermis, as well as inflammation in the dermis, which was not evident in control mice (Supplementary Fig. 3). Interestingly, erythema has been previously reported in mice treated with chemically unrelated RAF inhibitors ${ }^{6}$. Immunohistochemistry of serial skin sections showed increased staining for the proliferation marker Ki67 in the keratinocyte compartment of sections from inhibitor- but not vehicle-treated animals, co-localized with increased cytoplasmic phospho-ERK staining (Fig. 1c), which is intriguing in light of the recent clinical reports of
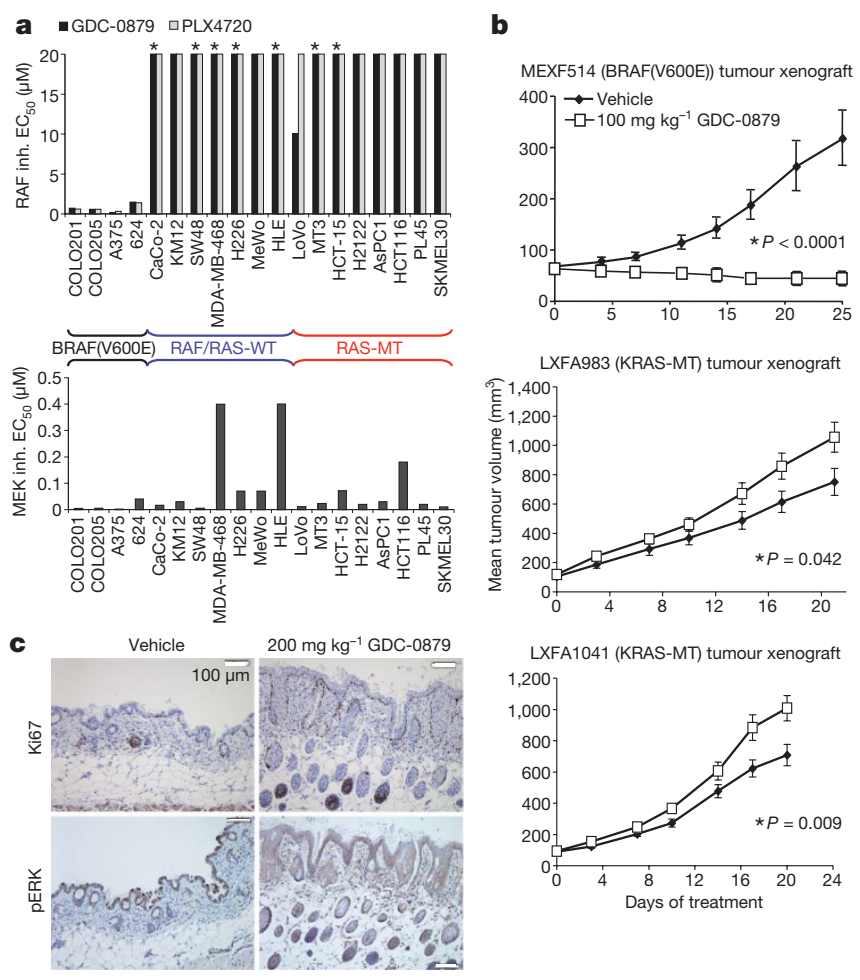

Figure 1 | RAF inhibitors are selective in inhibiting growth and proliferation of BRAF(V600E) lines. a, Half-maximum effective concentration $\left(\mathrm{EC}_{50}\right)$ values $(\mu \mathrm{M})$ in cellular proliferation assays of RAF inhibitors (inh.) (GDC0879 and PLX4720) and MEK inhibitor (PD0325901) against a panel of BRAF(V600E), RAS/RAF-WT and RAS-MT cell lines. Cell lines with hyperproliferation are noted with asterisks. $\mathbf{b}$, Tumour volume measurements of MEXF514 (BRAF(V600E)), LXFA983 (KRAS-MT) and LXFA1041 (KRAS-MT) xenograft tumours treated with vehicle or $100 \mathrm{mg} \mathrm{kg}^{-1}$ GDC-0879 once a day. Error bars represent s.e.m. with $n=10$. $P$ values were obtained by Dunnett's $t$-test. c, Ki67 (top) and phospho-ERK (pERK, bottom) staining of skin sections from mice treated with vehicle or $200 \mathrm{mg} \mathrm{kg}^{-1}$ GDC-0879 once per day for 21 days. All scale bars, $100 \mu \mathrm{m}$. 
cutaneous squamous cell carcinoma and keratoacanthoma for two selective RAF inhibitors ${ }^{7-9}$.

RAF inhibitor treatment of tumour cell lines in vitro resulted in the induction of phospho-MEK and phospho-ERK levels in the RAS/RAFWT (MeWo) and KRAS-MT (H2122) lines, versus their sustained inhibition in BRAF(V600E) (A375) cells (Fig. 2a). Knockdown of CRAF (also known as RAF1), but not BRAF, in HCT116 (KRASMT) cells ${ }^{10}$ was able to reverse the phospho-MEK induction observed after RAF inhibitor treatment, indicating that CRAF has the major role in signalling to MEK (Fig. 2b). Notably, both BRAF and CRAF kinase activities increased in a dose-dependent manner after GDC0879 treatment, selectively in non-BRAF(V600E) lines (Fig. 2c). The PLX4720 RAF inhibitor showed more modest effects, inducing moderate CRAF activity at a high concentration. Activation of ARAF was also observed selectively after GDC-0879 treatment, and dual $A R A F$ and CRAF knockdown was synergistic in decreasing inhibitorinduced phospho-MEK levels in HCT116 (KRAS-MT) cells (Supplementary Fig. 5).

RAF activation proceeds through the formation of homo- and heterodimers $^{11-13}$. The RAF inhibitor GDC-0879 induced B-C and $\mathrm{A}-\mathrm{B}$ heterodimers together with the induction of $\mathrm{A}-, \mathrm{B}-$ and CRAF kinase activities (Fig. 2c and Supplementary Figs 5 and 6). Because RAF heterodimers are expected to have higher specific kinase activity ${ }^{12}$
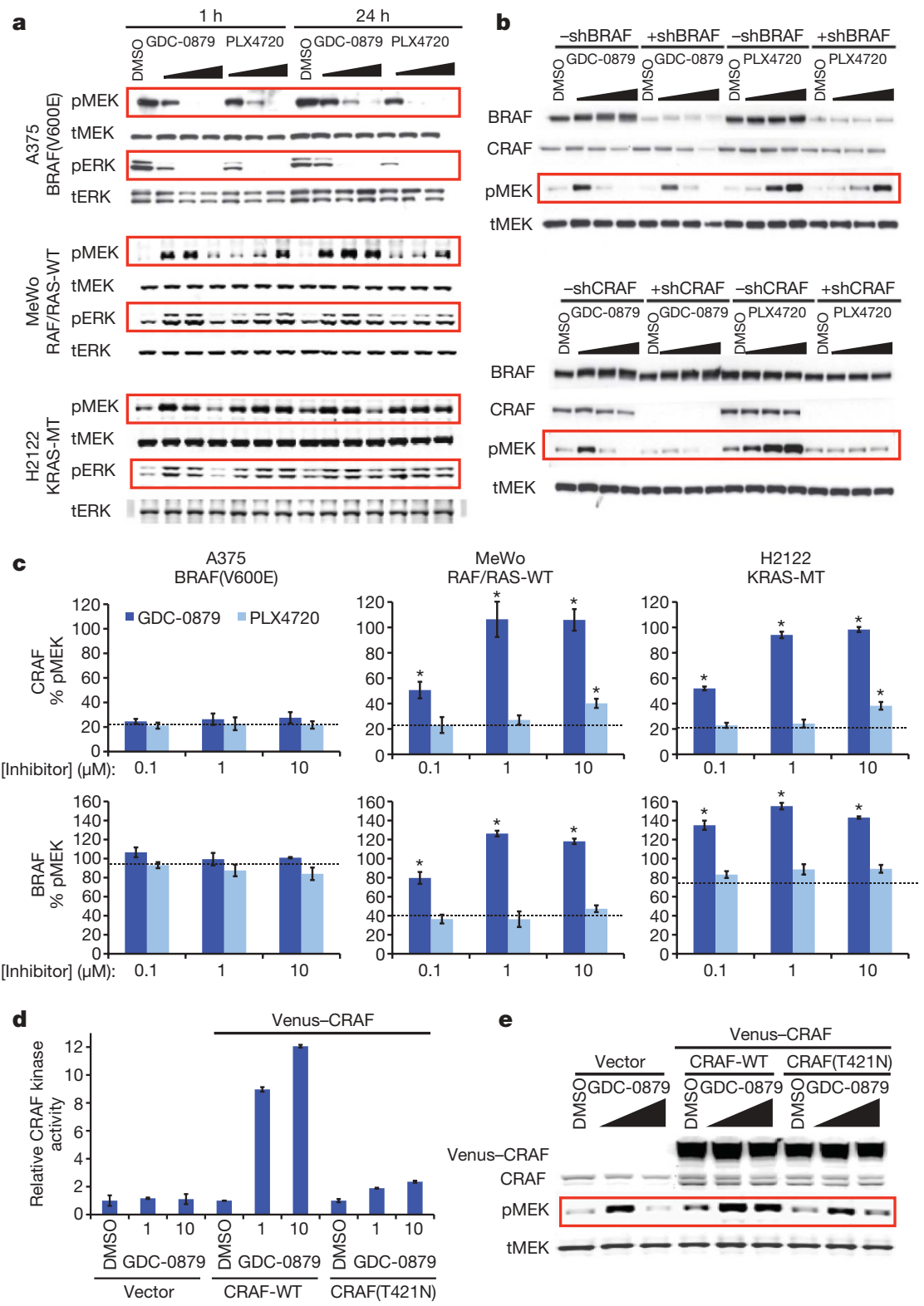

e

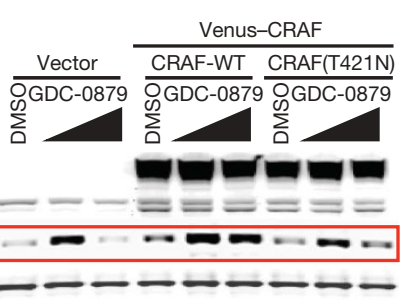

and low activity BRAF mutants can cause CRAF activation through heterodimerization ${ }^{11,14}$, we tested whether BRAF is required for CRAF activation and MEK phosphorylation. Treatment of isogenic $B R A F^{+/+}$and $B R A F^{-1-}$ cell lines ${ }^{15,16}$ with RAF inhibitors resulted in a similar induction of CRAF specific activity as well as phospho-MEK and phospho-ERK levels, demonstrating that BRAF is not essential (Supplementary Figs 7 and 8). Notably, in addition to heterodimers, RAF inhibitors induced CRAF homodimers (Supplementary Fig. 7c), providing a further mechanism for priming of CRAF activity ${ }^{17}$ when other isoforms are not expressed or heterodimer formation is impaired, as is the case for PLX4720 treatment. Kinase activation of CRAF(T421N), a gatekeeper mutant that does not bind either GDC0879 or PLX4720, was markedly impaired and phospho-MEK induction after RAF inhibitor treatment in CRAF(T421N) transfectants was similar to untransfected controls, demonstrating that inhibitor binding to the CRAF nucleotide-binding pocket is required for effective CRAF activation and downstream signalling (Fig. 2d, e and Supplementary Fig. 9b, c).

To address the question of whether CRAF kinase activity was required for phospho-MEK induction by RAF inhibitors, we characterized the chemically unrelated ATP-competitive RAF inhibitor AZ628 (ref. 18), which binds to the inactive conformation of the RAF kinase active site motif Asp-Phe-Gly (DFG-out conformation), has
Figure 2 | RAF inhibitors activate the MAPK pathway in non-BRAF(V600E) cells in a CRAFdependent manner. a, Immunoblot of lysates from A375, MeWo and $\mathrm{H} 2122$ cells treated with $0.1,1$ and $10 \mu \mathrm{M}$ GDC-0879 or PLX4720. pMEK (and pERK) refer to phospho-MEK (and -ERK) levels, whereas tMEK (and tERK) refer to total MEK (and ERK) levels. b, Immunoblot of lysates from HCT116 (KRAS-MT) cells carrying doxycycline (dox)-inducible short hairpin RNA (shRNA) directed against BRAF or CRAF. Cells were treated with $0.1,1$ and $10 \mu \mathrm{M}$ inhibitors for $1 \mathrm{~h}$ in the absence (-shRNA) or presence (+ shRNA) of dox. c, CRAF and BRAF immunoprecipitation kinase assays from lysates of A375, MeWo and $\mathrm{H} 2122$ cells treated with inhibitor for $1 \mathrm{~h}$. Error bars represent s.d. with $n=3 ;{ }^{*} P<0.001$ using a Student's $t$-test. d, e, HCT116 cells transiently transfected with control vector, Venus-CRAF-WT or Venus-CRAF(T421N) were treated with 1 and $10 \mu \mathrm{M}$ GDC-0879 for $1 \mathrm{~h}$. d, Venus-tagged CRAF immunoprecipitation kinase assay (data normalized to DMSO control with error bars representing s.d. of $n=3$ ). e, Immunoblot of lysates detecting endogenous CRAF,

Venus-CRAF and phospho-MEK. Data are representative of at least two independent experiments. 
high potency against CRAF and a very slow/irreversible off-rate (Supplementary Figs 10 and 12). We proposed that, when primed CRAF is blocked through prolonged binding of a potent RAF inhibitor to its active site, there should be no MEK phosphorylation if CRAF kinase activity is required. Indeed, CRAF complexes purified from AZ-628-treated cells show no in vitro kinase activity, and AZ-628treated cells show no phospho-MEK induction or hyperproliferation (Supplementary Figs 2 and 10d-f). In comparison, both GDC-0879 and PLX4720 show very rapid off-rates and cannot persistently occupy the ATP-binding pocket to effectively block CRAF kinase activity and phospho-MEK induction (Supplementary Fig. 10). This series of experiments suggests that CRAF kinase activity is required for both phospho-MEK induction and cellular hyper-proliferation.

To gain a better understanding of the RAF activation sequence in response to inhibitor priming, we characterized the topology of the events and the role of RAS-GTP. Fractionation experiments demonstrated that all RAF inhibitors tested, except PLX4720, induced a dose-dependent translocation to the membrane fraction of BRAF and CRAF, accompanied by increased CRAF(S338) phosphorylation $^{19}$, selectively in non-BRAF(V600E) lines (Fig. 3a and Supplementary Fig. 11). Membrane targeting was RAS-GTP dependent, because expression of dominant-negative KRAS(S17N) led to cytoplasmic retention of CRAF after inhibitor treatment, versus the membrane localization observed with KRAS-WT (Fig. 3b). RAF inhibitors induce membrane and/or cytosolic phospho-MEK levels, together with their effects on CRAF localization, with the exception of AZ-628 as previously discussed (Fig. 3a and Supplementary Figs 10 and 11). After KRAS(S17N) transfection, the induction of both membrane and cytosolic phospho-MEK levels is significantly impaired (Supplementary Fig. 11b). Thus, as a consequence of inhibitor binding, CRAF gets activated in a RAS-GTP-dependent manner, through membrane translocation, Ser 338 phosphorylation and dimerization $^{20-22}$. The important role of RAS-GTP is further underscored by the fact that transfection of BRAF(V600E) A375 cells with activated mutant KRAS(G12D), but not wild-type KRAS, leads to CRAF-BRAF heterodimerization and CRAF kinase activation after inhibitor treatment (Supplementary Fig. 11d).

The conformation of the ATP-binding pocket is crucial for kinase maturation and has an important role in the ability of small molecules to prime and induce the translocation of their target kinases to the membrane ${ }^{23-25}$. Membrane targeting of CRAF requires inhibitor binding to the ATP-binding pocket, because the gatekeeper threonine mutant CRAF(T421N) (which does not bind to GDC-0879 and weakly to AZ-628; Supplementary Fig. 9a) remains in the cytoplasm after inhibitor treatment (Fig. 3c). Furthermore, membrane targeting of CRAF does not seem to require kinase activity, as CRAF(D486A), a DFG-loop aspartate mutant devoid of kinase activity, is still able to translocate to the membrane (Fig. $3 \mathrm{c}$ ) as long as it can bind the inhibitor, as is the case for AZ-628 but not GDC-0879 (Supplementary Fig. 12a). Thus, membrane targeting is not due to CRAF kinase inhibition and does not proceed through an extrinsic mechanism, such as feedback, but instead relies on inhibitor occupancy of the ATP-binding pocket.

To test further whether priming was mediated by the inhibitors' conformational effects on the RAF kinase domain, we assayed BRAFCRAF heterodimerization biochemically. The CRAF kinase domain a

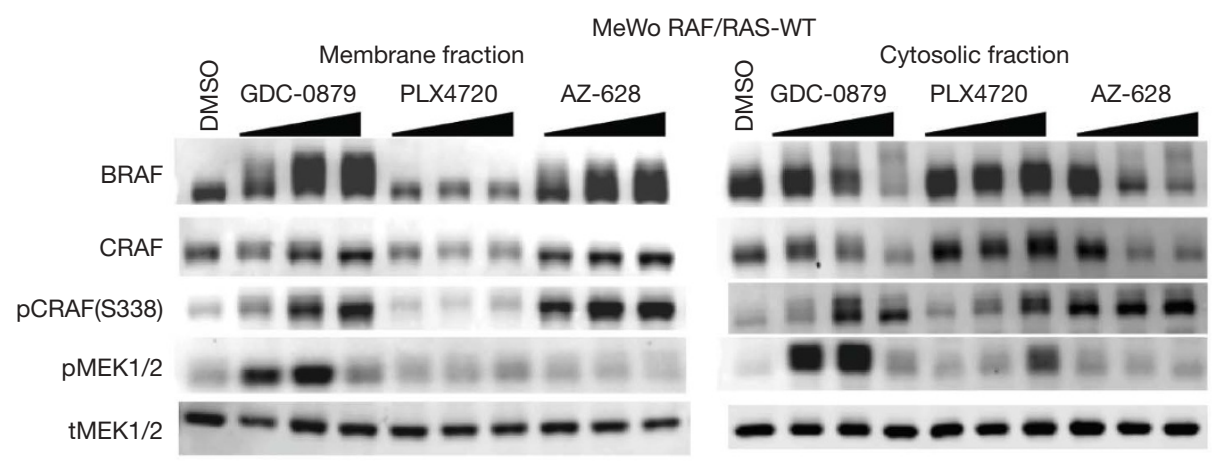

b
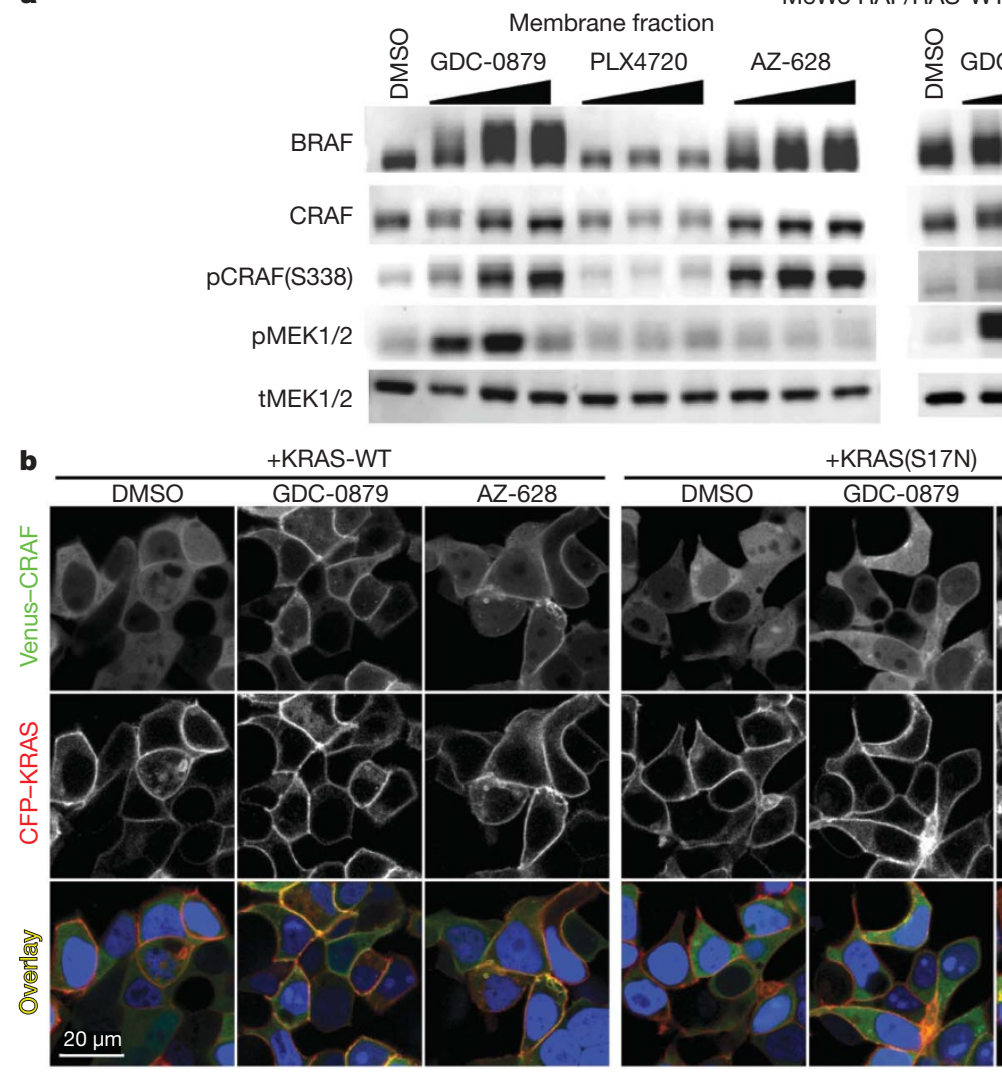

c

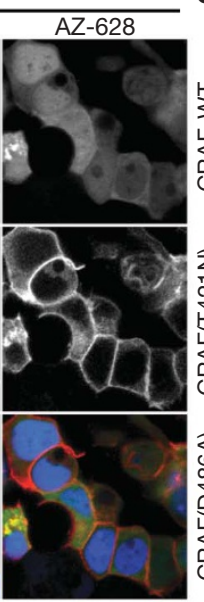

C

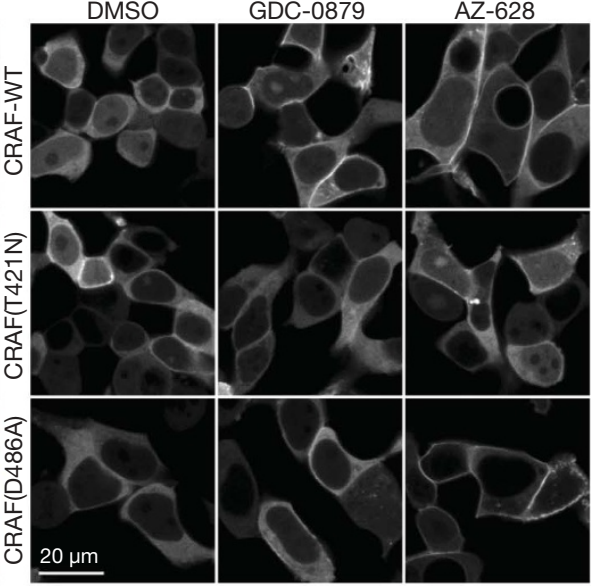

Figure 3 | RAF inhibitors induce RAS-dependent translocation of wild-type RAF to the plasma membrane in non-BRAF(V600E) cells. a, Membrane (P100) and cytosolic (S100) fractionation of MeWo cells treated with inhibitors at $0.1,1$ and $10 \mu \mathrm{M}$ for $1 \mathrm{~h}$ and immunoblotted with the indicated antibodies. pMEK1/2 refers to phospho- and tMEK1/2 refers to total MEK1/ 2 levels, respectively. b, HEK293T cells were transiently transfected with Venus-CRAF (green), CFP-KRAS (red) and mCherry-H2B (blue). Venustagged CRAF co-localizes with CFP-KRAS on the plasma membrane in cells treated with $10 \mu \mathrm{M}$ GDC-0879 or AZ-628 for $4 \mathrm{~h}$. Membrane translocation is blocked when the dominant-negative CFP-tagged $\mathrm{KRAS}(\mathrm{S} 17 \mathrm{~N})$ is transfected instead of KRAS-WT. c, Live cell imaging of Venus-tagged CRAF-WT, CRAF(T421N) and CRAF(D486A) co-transfected with CFP-KRAS in HEK293T cells. Cells were treated with $10 \mu \mathrm{M}$ GDC-0879 or AZ-628 for $4 \mathrm{~h}$. Images are representative of three independent experiments performed in triplicate. 
forms a stable complex with the BRAF kinase domain in the absence of any inhibitor in an immunoprecipitation and western blot assay using purified recombinant proteins (Fig. 4a). Notably, both a non-hydrolysable analogue of ATP and PLX4720 destabilize, whereas AZ-628 and GDC-0879 significantly stabilize, the CRAF-BRAF heterodimer kinase domain interaction (Fig. 4a). This result is consistent with our findings from the cellular immunoprecipitation and western blot experiments in non-BRAF(V600E) cell lines (Supplementary Figs 6 and 10), and supports the model that dimerization is mediated directly through the inhibitors' effects on the RAF kinase domain, rather than being a secondary effect. The inhibitors had no effect on the basal interaction between the CRAF and BRAF(V600E) kinase domains (Fig. 4a), in agreement with the cellular data demonstrating lack of priming in BRAF(V600E) mutant lines (Fig. 2 and Supplementary Fig. 6). In summary, our biochemical and cellular data suggests that inhibitor occupancy of the ATP-binding pocket can effect RAF dimerization and that the inhibitors' effects are different from ATP itself.

To probe further the structural basis of the inhibitors' effects on CRAF we solved the crystal structure of the CRAF kinase domain complexed to a close analogue of GDC-0879 (ref. 4) (Supplementary Fig. 13 and Supplementary Table 1). The CRAF-inhibitor complex adopts a dimer conformation in the asymmetric unit, as previously described for $\mathrm{BRAF}^{26}$. Comparison of the CRAF and BRAF homodimers shows an extremely well-conserved interface, the single difference among them residing in the most amino-terminal residue of CRAF resolved in the crystal structure, CRAF(Y340), within its negative-charged regulatory region ${ }^{27}$ (Fig. $4 \mathrm{~b}$ and Supplementary Table 2). Further amino acid residues that differ between BRAF and CRAF are highlighted in Fig. 4b. A heterodimer model of BRAFCRAF illustrates that, in contrast to the conformation induced by
GDC-0879 and AZ-628 when bound to BRAF, PLX4720 induces a shift in the $\alpha$ C-helix in BRAF (cyan), bringing it closer to the dimer interface, which may affect priming (Fig. 4c).

Finally, to demonstrate formally that modulation of kinase domain dimerization by ATP-competitive RAF inhibitors underlies their ability to activate CRAF and downstream signalling to MEK, we generated CRAF and BRAF dimer interface mutants that either constitutively heterodimerize $(\mathrm{CRAF}(\mathrm{E} 478 \mathrm{~K})$ and $\mathrm{BRAF}(\mathrm{E} 586 \mathrm{~K}))$ or are defective in dimerization $(\mathrm{CRAF}(\mathrm{R} 401 \mathrm{H}) \text { and } \mathrm{BRAF}(\mathrm{R} 509 \mathrm{H}))^{26}$. Cotransfection of CRAF(E478K) and BRAF(E586K) in HCT116 KRASMT cells resulted in a basal increase of exogenous CRAF kinase activity compared to wild-type controls (Fig. 4d). No further induction was observed after RAF inhibitor treatment, confirming that dimerization is the main driver for the inhibitors' effects on CRAF kinase activity. Constitutive heterodimerization by these mutants was confirmed (Fig. 4d), suggesting that the interactions characterized by our assays were mediated at the level of kinase domain dimer interface. In contrast, co-transfection of CRAF(R401H) and BRAF(R509H) lead to decreased levels of basal CRAF activity and a significant impairment of CRAF activation by RAF inhibitors, consistent with the mutants' defective heterodimerization properties (Fig. 4d). Phospho-MEK level induction by RAF inhibitors correlated with the efficiency of heterodimer formation and CRAF activation in all cases (Supplementary Fig. 15).

In conclusion, we have discovered that ATP-competitive RAF inhibitors have opposing roles as inhibitors and activators of the RAFMEK-ERK pathway, and that the outcome is dependent on cellular context and genotype. In BRAF(V600E) cells, the pathway is sensitized to ATP-competitive RAF inhibitors owing to the higher ATP apparent $K_{\mathrm{m}}\left(K_{\mathrm{m}(\mathrm{app})}\right)$ of the mutant BRAF(V600E) protein (Supplementary Fig. 4) and lack of CRAF activation (Supplementary Fig. 1). In a
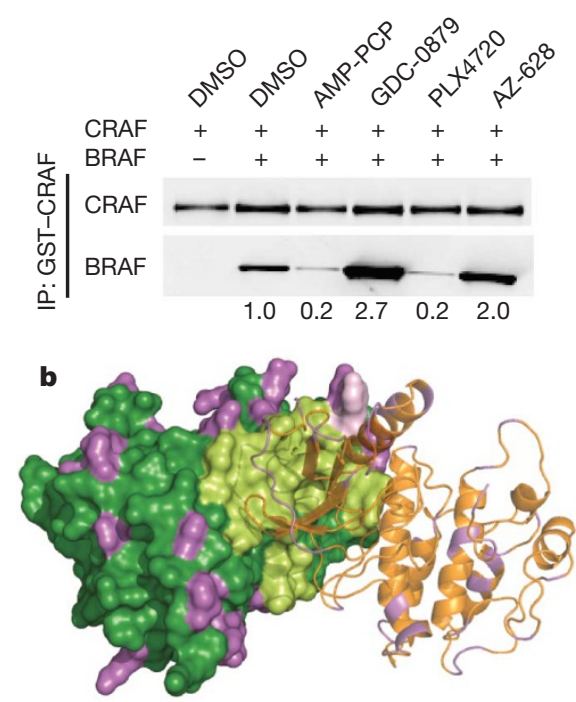

CRAF-KD-BRAF(V600E)-KD heterodimer
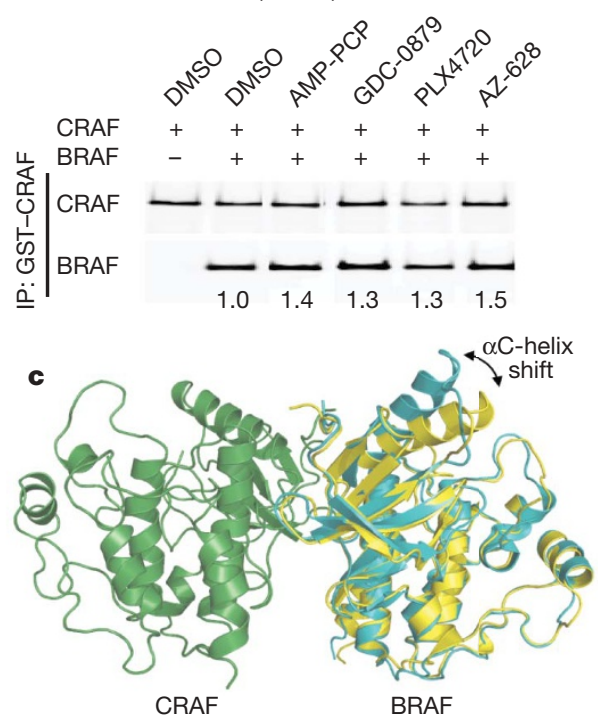

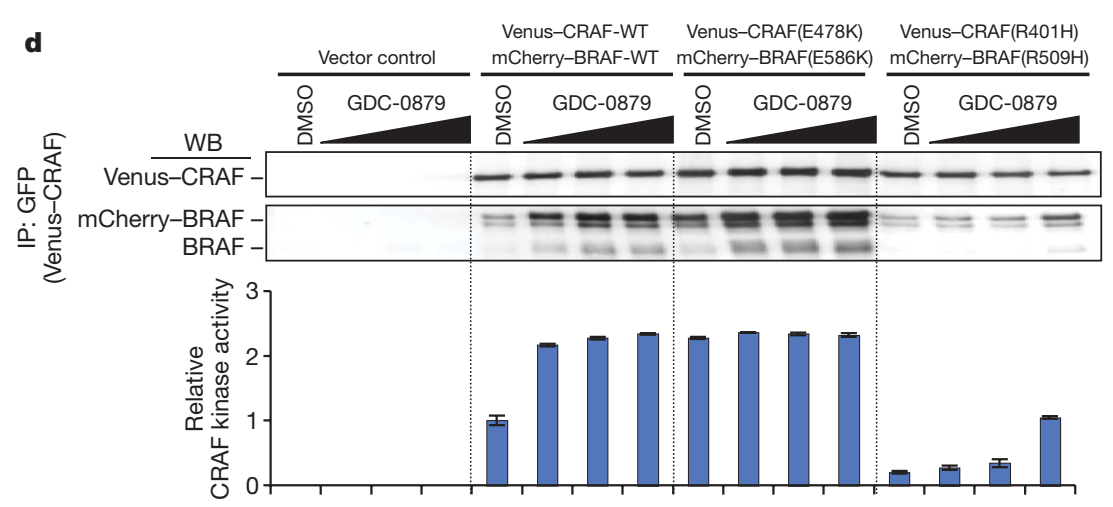

Figure 4 | Modulation of kinase domain dimerization by ATP-competitive RAF inhibitors underlies their ability to activate CRAF.

a, Biochemical dimerization assays (immunoprecipitation (IP) and western blot) using recombinant RAF proteins in the presence of DMSO, $10 \mu \mathrm{M}$ inhibitors or $1 \mathrm{mM}$ AMP-PCP. Bands were quantified and BRAF levels were normalized to captured GST-CRAF. KD, kinase domain. b, CRAF crystal structure with a GDC0879 analogue ${ }^{4}$ reveals a side-to-side dimer. Nonconserved residues between BRAF and CRAF are coloured in violet. Conserved residues in the surface depiction are shown in green; residues in the dimer interface are shown in lime green. c, BRAF-CRAF heterodimer model derived from CRAF-GDC-0879 analogue (green) and BRAF-GDC-0879 analogue (yellow) or BRAF-PLX4720 (cyan). d, HCT116 cells were transfected with the indicated wild-type and dimerization mutant RAF constructs for $48 \mathrm{~h}$ and treated with $0.1,1$ and $10 \mu \mathrm{M}$ GDC- 0879 for $1 \mathrm{~h}$. Immunoprecipitation and western blot (WB) for Venus-CRAF and associated BRAF (top) and wild-type or mutant Venus-CRAF immunoprecipitation kinase assay (bottom). Error bars represent s.d. of $n=3$. Data are representative of two independent experiments. 
BRAF-WT cells, inhibitors trigger the CRAF activation sequence, which can lead to MEK/ERK phosphorylation and in some cases to enhanced growth (Supplementary Fig. 1). This suggests that careful assessment should be made of the functional consequences of other ATP-competitive inhibitors with respect to priming, which may affect the inhibitors' efficacy and safety profiles. On the basis of our findings, we propose that preventing activation of CRAF could potentially prevent undesirable effects of RAF inhibitors on normal tissue and nonBRAF(V600E) tumours. This could be attained by an allosteric nonATP competitive mechanism that would prevent RAF dimerization, or RAS-RAF RAS-binding domain (RBD) interaction. This data also highlights the need for BRAF(V600E) patient selection in V600Eselective RAF inhibitor clinical trials.

\section{METHODS SUMMARY}

Chemicals and cell culture. PLX4720 and PD0325901 were purchased from Symansis, and EMD from CalBiochem. AZ-628 was synthesized according to the procedure described in patent number WO2006024834 (http://www.wipo. int/pctdb/en/wo.jsp?wo=2006024834).

Fluorescence microscopy. For CRAF sub-cellular localization experiments, HEK293T cells were transiently transfected with relevant cyan fluorescent protein (CFP)-KRAS and Venus-CRAF constructs and TagBFP or mCherry$\mathrm{H} 2 \mathrm{~B}$ to identify nuclei. Compounds were added $20 \mathrm{~h}$ after transfection and live cells were imaged $24 \mathrm{~h}$ after transfection.

Tumour xenograft studies and immunohistochemistry. Tumour fragments were obtained from MEXF514, LXFA983 and LXFA1041 xenografts. In vivo studies were conducted at Oncotest. Immunohistochemistry were conducted as described previously ${ }^{3}$.

In vitro kinase assays. Cell lysates were immunoprecipitated with anti-CRAF, anti-BRAF or anti-GFP antibody (Millipore) and then incubated with $0.4 \mu \mathrm{g}$ unactive MEK1 (Millipore) in $40 \mu$ of kinase buffer ( $20 \mathrm{mM}$ MOPS, pH 7.2, $25 \mathrm{mM} \beta$-glycerol phosphate, $5 \mathrm{mM}$ EGTA, $1 \mathrm{mM}$ sodium orthovanadate, $1 \mathrm{mM}$ dithiothreitol (DTT), $120 \mu \mathrm{M}$ ATP, $18 \mathrm{mM} \mathrm{MgCl}_{2}$ ) for $30 \mathrm{~min}$ at $30^{\circ} \mathrm{C}$. Samples were then loaded onto the Meso Scale Discovery Phospho (Ser 217/ 221)/Total MEK1/2 assay plates and quantified.

Expression and crystallization of CRAF. The dual expression baculovirus construct pBac4x-1-HTH-CRAF(324-618):CDC37 was expressed in Hi5 cells. CRAF was co-crystallized with inhibitor in hanging drops (12\% PEG $8 \mathrm{~K}$, $100 \mathrm{mM}$ Tris, $\mathrm{pH} 8.0,10 \%$ tacsimate, grown at $12{ }^{\circ} \mathrm{C}$ ).

RAF dimerization. His-BRAF-WT kinase domain or His-BRAF(V600E) kinase domain and glutathione $S$-transferase (GST)-CRAF kinase domain (Invitrogen) were incubated in the presence of a fixed concentration of compound or dimethylsulphoxide (DMSO) for $1 \mathrm{~h}$ and immunoprecipitated with a rabbit anti-GST antibody.

Full Methods and any associated references are available in the online version of the paper at www.nature.com/nature.

\section{Received 29 September 2009; accepted 18 January 2010.}

Published online 3 February 2010.

1. Schubbert, S., Shannon, K. \& Bollag, G. Hyperactive Ras in developmental disorders and cancer. Nature Rev. Cancer 7, 295-308 (2007).

2. Yeh, J. J. et al. KRAS/BRAF mutation status and ERK1/2 activation as biomarkers for MEK1/2 inhibitor therapy in colorectal cancer. Mol. Cancer Ther. 8, 834-843 (2009).

3. Hoeflich, K. P. et al. Antitumor efficacy of the novel RAF inhibitor GDC-0879 is predicted by BRAF ${ }^{\mathrm{V} 600 \mathrm{E}}$ mutational status and sustained extracellular signalregulated kinase/mitogen-activated protein kinase pathway suppression. Cancer Res. 69, 3042-3051 (2009).

4. Hansen, J. D. et al. Potent and selective pyrazole-based inhibitors of B-Raf kinase. Bioorg. Med. Chem. Lett. 18, 4692-4695 (2008).

5. Tsai, J. et al. Discovery of a selective inhibitor of oncogenic B-Raf kinase with potent antimelanoma activity. Proc. Natl Acad. Sci. USA 105, 3041-3046 (2008).

6. Malek, S. Selective inhibition of Raf results in downregulation of the RAS/RAF/ MEK/ERK pathway and inhibition of tumor growth in vivo. Eur. J. Cancer, Suppl. 4, 184 (2006)
7. Flaherty, K. et al. Phase I study of PLX4032: proof of concept for V600E BRAF mutation as a therapeutic target in human cancer. J. Clin. Oncol. 27, abstract 9000 (2009).

8. Schwartz, G. K. et al. A phase I study of XL281, a selective oral RAF kinase inhibitor, in patients (Pts) with advanced solid tumors. J. Clin. Oncol. 27, abstract 3513 (2009).

9. Chapman, P. et al. Early efficacy signal demonstrated in advanced melanoma in a phase I trial of the oncogenic BRAF-selective inhibitor PLX4032. Eur. J. Cancer, Suppl. 7, 5 (2009).

10. Jaiswal, B. S. et al. Combined targeting of BRAF and CRAF or BRAF and PI3K effector pathways is required for efficacy in NRAS mutant tumors. PLoS One 4, e5717 (2009).

11. Garnett, M. J. et al. Wild-type and mutant B-RAF activate C-RAF through distinct mechanisms involving heterodimerization. Mol. Cell 20, 963-969 (2005).

12. Rushworth, L. K., Hindley, A. D., O'Neill, E. \& Kolch, W. Regulation and role of Raf1/B-Raf heterodimerization. Mol. Cell. Biol. 26, 2262-2272 (2006).

13. Weber, C. K., Slupsky, J. R., Kalmes, H. A. \& Rapp, U. R. Active Ras induces heterodimerization of cRaf and BRaf. Cancer Res. 61, 3595-3598 (2001).

14. Wan, P. T. et al. Mechanism of activation of the RAF-ERK signaling pathway by oncogenic mutations of B-RAF. Cell 116, 855-867 (2004)

15. Kim, J. S., Lee, C., Foxworth, A. \& Waldman, T. B-Raf is dispensable for K-Rasmediated oncogenesis in human cancer cells. Cancer Res. 64, 1932-1937 (2004).

16. Yun, J. et al. Glucose deprivation contributes to the development of KRAS pathway mutations in tumor cells. Science 325, 1555-1559 (2009).

17. Zang, M. et al. Characterization of $\mathrm{Ser}^{338}$ phosphorylation for Raf-1 activation. J. Biol. Chem. 283, 31429-31437 (2008).

18. Khazak, V., Astsaturov, I., Serebriiskii, I. G. \& Golemis, E. A. Selective Raf inhibition in cancer therapy. Expert Opin. Ther. Targets 11, 1587-1609 (2007).

19. Goetz, C. A., O'Neil, J. J. \& Farrar, M. A. Membrane localization, oligomerization, and phosphorylation are required for optimal raf activation. J. Biol. Chem. 278, 51184-51189 (2003).

20. Xiang, $X$. et al. Phosphorylation of 338SSYY341 regulates specific interaction between Raf-1 and MEK1. J. Biol. Chem. 277, 44996-45003 (2002).

21. Terai, K. \& Matsuda, M. Ras binding opens c-Raf to expose the docking site for mitogen-activated protein kinase kinase. EMBO Rep. 6, 251-255 (2005).

22. Jelinek, T. et al. RAS and RAF-1 form a signalling complex with MEK-1 but not MEK-2. Mol. Cell. Biol. 14, 8212-8218 (1994).

23. Cameron, A. J. et al. PKC maturation is promoted by nucleotide pocket occupation independently of intrinsic kinase activity. Nature Struct. Mol. Biol. 16, 624-630 (2009).

24. Okuzumi, T. et al. Inhibitor hijacking of Akt activation. Nature Chem. Biol. 5, 484-493 (2009).

25. Hindie, V. et al. Structure and allosteric effects of low-molecular-weight activators on the protein kinase PDK1. Nature Chem. Biol. 5, 758-764 (2009).

26. Rajakulendran, T. et al. A dimerization-dependent mechanism drives RAF catalytic activation. Nature 461, 542-545 (2009).

27. Wellbrock, C., Karasarides, M. \& Marais, R. The RAF proteins take centre stage. Nature Rev. Mol. Cell Biol. 5, 875-885 (2004)

Supplementary Information is linked to the online version of the paper at www.nature.com/nature.

Acknowledgements We would like to thank the chemists at Genentech and Array BioPharma for providing GDC-0879. We thank Oncotest GmbH, L. Murray, S. Gould, B. Alicke, W. Voegtli, K. Bock, H. Stern and the immunohistochemistry core laboratory for technical support. We thank B. Vogelstein for HCT116 isogenic $B R A F^{+/+}$and $B R A F^{-/-}$cell lines, and T. Waldman for the HEC1A BRAF $F^{+/+}$and $B R A F^{-/-}$cell lines. Special thanks goes to $A$. Bruce for help with artwork.

Author Contributions G.H. and S.M. designed the studies, interpreted the data and wrote the manuscript. K.S., I.Y., B.L., S.S. and D.S. conducted cellular experiments and dimerization assays. D.J.A., M.J.C.L. and R.A. conducted microscopy experiments. B.J.B., G.V., T.M. and I.A. conducted crystallography and provided structural input. S.L.G. conducted enzymology. K.P.H. and H.K. conducted in vivo experiments and immunohistochemistry. B.S.J. and S.S. generated inducible shRNA cell lines. M.B. and L.S.F. interpreted the data and wrote the manuscript.

Author Information Atomic and structural data are deposited in Protein Data Bank under accession number 3LB7. Reprints and permissions information is available at www.nature.com/reprints. The authors declare competing financial interests: details accompany the full-text HTML version of the paper at www.nature.com/ nature. Correspondence and requests for materials should be addressed to G.H. (hatzivassiliou.georgia@gene.com) or S.M. (malek.shiva@gene.com). 


\section{METHODS}

Chemicals and cell culture. PLX4720 and PD0325901 were purchased from Symansis, and EMD from CalBiochem. AZ-628 was synthesized according to the procedure described in patent number WO2006024834 (http://www.wipo. $\mathrm{int} / \mathrm{pctdb} / \mathrm{en} / \mathrm{wo} . \mathrm{jsp}$ ?wo $=2006024834)$. All cells lines were obtained from the American Type Culture Collection (ATCC) except for the isogenic pairs of cell lines. Hecla $B R A F^{+/+}$and Hecla $B R A F^{-1-}$ were obtained from T. Waldman's laboratory. HCT116 $B R A F^{+/+}$and HCT116 $B R A F^{-1-}$ were obtained from Horizon Discovery.

Enzymology assays. Full-length $6 \times$ His-tagged human BRAF(V600E) coexpressed with human CDC37 (1-378), full-length $6 \times$ His tagged human BRAF-WT, and full-length Flag-tagged human wild-type CRAF were expressed in baculovirus-infected insect cells and purified using standard affinity tag chromatographic methods. The enzymatic activity of was quantified by measuring the incorporation of radiolabel from $\left[\gamma-{ }^{33} \mathrm{P}\right]$ ATP into full-length $6 \times$ His-tagged human MEK covalently modified with $5^{\prime}$-p-fluorosulphonylbenzoyladenosine (FSBA). Standard enzyme reaction conditions have been described previously ${ }^{28}$. ATP $K_{\mathrm{m}(\mathrm{app})}$ values were determined by monitoring the enzymatic activity over 120 min at a constant amount of $\left[\gamma^{-33} \mathrm{P}\right]$ ATP $\left(33 \mu \mathrm{Ci} \mathrm{ml}^{-1}\right)$, while varying ATP. ATP $K_{\mathrm{m}(\text { app })}$ values were determined by fitting a hyperbolic model to the enzymatic rate versus $[\mathrm{ATP}]$ data. Half-maximum inhibitory concentration $\left(\mathrm{IC}_{50}\right)$ values were calculated by fitting a standard four-parameter logistic model to the dose response curve plotted as the per cent of control versus concentration of compound. The apparent inhibition constant $\left(K_{\mathrm{i}(\mathrm{app})}\right)$ and predicted $\mathrm{IC}_{50}$ values at 1 mM ATP were calculated using the Cheng-Prusoff relationship ${ }^{29}$ assuming compounds are competitive with ATP. For off-rate experiments, BRAF(V600E) protein was pre-incubated with 100 -fold excess of enzyme and compound and the mixture was diluted 100-fold at the initiation of the kinase activity assay for BRAF(V600E). The inhibitor off-rates were calculated from measuring the rate of kinase activity rebound of the inhibitor-preincubated BRAF(V600E) compared to non-preincubated control.

Fluorescent protein-fusion constructs. KRAS, BRAF, CRAF and H2B were amino-terminally tagged with fluorescent proteins using multisite Gateway cloning (Invitrogen). Relevant point mutations were introduced using sitedirected mutagenesis. Each fusion was inserted into the CMV-driven pcDNADEST vector, in which the carboxy-terminal GFP tag was not translated owing to a stop codon following the inserted genes. Cells were transfected with Lipofectamine2000 (Invitrogen), according to the manufacturer's instructions. Microscopy. For CRAF sub-cellular localization experiments, HEK293T cells were transiently transfected with relevant CFP-KRAS and Venus-CRAF constructs and TagBFP or mCherry-H2B (Clontech) ${ }^{30,31}$ to identify nuclei. Compounds were added $20 \mathrm{~h}$ after transfection and live cells were imaged $24 \mathrm{~h}$ after transfection using an SP5 point-scanning confocal microscope with a $\times 40$ 1.25 numerical aperture (NA) HCX PL APO CS oil-immersion objective (Leica Microsystems).

Tumour xenograft studies and immunohistochemistry. Tumour fragments obtained from MEXF514, LXFA983 and LXFA1041 xenografts (conducted at Oncotest) were cut into $1-2-\mathrm{mm}^{3}$ pieces and implanted subcutaneously into NMRI nu/nu mice (Taconic). GDC-0879 compound was prepared fresh weekly in $0.5 \%$ methylcellulose and $0.2 \%$ Tween- 80 in water, stored at $4{ }^{\circ} \mathrm{C}$, and administered daily by oral gavage. Tumours were staged and measured as described previously ${ }^{3}$. Xenograft tissues were fixed for $24 \mathrm{~h}$ in $10 \%$ neutral buffered formalin and were then processed and paraffin embedded. Immunohistochemistry was conducted as described previously ${ }^{3}$.

Cell viability assays. Cell viability assays were carried out as described previously ${ }^{3}$. In brief, cells were seeded at 2,000 per well and treated with compound on day 2 . The relative numbers of viable cells were measured by luminescence using CellTiter-Glo (Promega).

Membrane fractionation. Cells were lysed in buffer A (25 mM Tris, pH 7.5, 1 mM EDTA, $1 \mathrm{mM}$ DTT, $10 \mathrm{mM} \mathrm{NaCl}, 25 \mathrm{mM} \mathrm{NaF}$, protease inhibitors and phosphatase inhibitors) and mechanically disrupted using a Dounce homogenizer. Cytosolic (S100) and membrane (P100) fractions were separated as described previously $^{32}$.

Immunoblotting. Cells lysed in lysis buffer (0.5\% NP40, $20 \mathrm{mM}$ Tris, pH 7.5, $137 \mathrm{mM} \mathrm{NaCl}, 10 \%$ glycerol, $1 \mathrm{mM}$ EDTA) plus protease inhibitor mixturecomplete mini (Roche Applied Science) and phosphatase inhibitor mix (Pierce). Ten-to-twenty micrograms of protein from each sample was separated by SDS-PAGE and transferred to nitrocellulose membranes. Membranes were incubated with the indicated primary antibodies and analysed either by the enhanced chemiluminescence (ECL) detection system (Amersham) or by the addition of Alexa Fluor 647 goat anti-mouse (Molecular Probes Inc.).
In vitro kinase assays. Cells were plated and lysed as described in immunoblotting method. Cell lysates were incubated with anti-CRAF, anti-BRAF, or antiGFP antibody (Millipore) and $50 \mu \mathrm{l}$ of Protein A agarose beads (Millipore) for $2 \mathrm{~h}$ at $4{ }^{\circ} \mathrm{C}$. After washing with lysis buffer plus protease and phosphatase inhibitor mixtures, protein A beads were incubated with $0.4 \mu \mathrm{g}$ of unactive MEK1 (Millipore) in $40 \mu \mathrm{l}$ of kinase buffer (20 mM MOPS, pH 7.2, $25 \mathrm{mM} \beta$-glycerol phosphate, $5 \mathrm{mM}$ EGTA, $1 \mathrm{mM}$ sodium orthovanadate, $1 \mathrm{mM}$ DTT, $120 \mu \mathrm{M}$ ATP, $18 \mathrm{mM} \mathrm{MgCl}_{2}$ ) for $30 \mathrm{~min}$ at $30^{\circ} \mathrm{C}$. Samples were then loaded onto the Meso Scale Discovery Phospho (Ser 217/221)/Total MEK1/2 assay plates and quantified.

Expression and purification of CRAF for crystallization. The dual expression baculovirus construct pBac4x-1-HTH-CRAF(324-618): CDC37 was expressed in Hi5 cells. Baculovirus pellets were homogenized in $25 \mathrm{mM}$ Tris, $\mathrm{pH} 7.5,0.5 \mathrm{M}$ $\mathrm{NaCl}, 10 \%$ glycerol, $10 \mathrm{mM}$ imidazole, $10 \mathrm{mM} \beta$-mercaptoethanol, $0.035 \%$ Brij35 COMPLETE EDTA-free (Roche) and solubilized with a PANDA homogenizer. After centrifugation, the soluble fraction was mixed with Talon (Clontech) resin for $16 \mathrm{~h}$ at $4{ }^{\circ} \mathrm{C}$. The protein was eluted with an imidazole-containing buffer and applied to a Q Sepharose column, followed by a Mono S column and then loaded onto a Superdex 75 10/30 equilibrated with $25 \mathrm{mM}$ HEPES, pH 7.0, $0.3 \mathrm{M} \mathrm{NaCl}$, $15 \%$ glycerol, $0.25 \%$ CHAPS, 1 mM EDTA, 1 mM TCEP buffer. The monomeric CRAF pool was concentrated to $2.3 \mathrm{mg} \mathrm{ml}^{-1}$ for crystallization trials.

Crystallization and structure solution of CRAF. CRAF was co-crystallized with Inhibitor 18 (ref. 4) in hanging drops (12\% PEG 8K, 100 mM Tris, pH 8.0, 10\% tacsimate, grown at $12{ }^{\circ} \mathrm{C}$ ). Crystals were cryoprotected in 10\% PEG 8000, $10 \%$ tacsimate, $0.1 \mathrm{M}$ Tris, $\mathrm{pH} 8.0,25 \%$ glycerol and flash-frozen on an Oxford Cryosystems coldstage. The crystal was frozen at $100 \mathrm{~K}$ and data were collected in-house on an FR-E generator and Raxis-IV + + detector (Rigaku). The crystals diffracted to $4.0 \AA$. Diffraction data were processed with the CCP4 suite ${ }^{33}$. Molecular replacement searches were carried out with Molrep, using a previous structure of BRAF co-crystallized with Inhibitor 18 (Protein Data Bank (PDB) accession code $3 \mathrm{D} 4 \mathrm{Q}$ ). Molrep successfully found solutions for two monomers, in space group P41212. This gave a fractional occupancy 0.498 and no other monomers could be found. Because the crystal only diffracted to $4.0 \AA$, further precautions were taken during refinement in Refmac: non-crystallographic symmetry (NCS) restraints were enforced on all residues of both chains and a low $\mathrm{X}$-ray weighting term of 0.0025 was used. Also, all atoms were given a fixed isotropic B-factor or 68.0. No water atoms were added to the structure and, although there was weak density visible for the ligand, it was not modelled in. The final structure had reasonably good geometry (see Supplementary Table 1), and in the Ramachandran plot of the final structure only 8 out of 468 residues $(1.7 \%)$ are in disallowed regions.

Immunoprecipitation and western blots for dimerization. In assay buffer (25 mM HEPES, pH 7.4, $10 \mathrm{mM} \mathrm{MgCl}_{2}, 0.01 \%$ (v/v) Triton X-100, 2 mM DTT, $0.25 \%$ DMSO), $500 \mathrm{nM}$ of enzyme: Flag-CRAF full-length, His-BRAF-WT or B-RAF(V600E) kinase domain, or GST-CRAF kinase domain (Invitrogen) in the presence of a fixed concentration of compound or vehicle was incubated for $1 \mathrm{~h}$ at room temperature. AMP-PCP was purchased from Sigma. Proteins were immunoprecipitated with rabbit anti-Flag (Sigma, F7425) or rabbit anti-GST (Cell Signaling Technology, 2622) antibodies bound to protein A agarose beads (Millipore). Each sample was separated by SDS-PAGE and transferred to nitrocellulose membranes. Membranes were incubated with anti-CRAF (BD Biosciences, 610152) or anti-BRAF (Sigma, WH0000673M1) primary antibodies, and analysed by the addition of a secondary Alexa Fluor 488 goat anti-mouse IgG $(\mathrm{H}+\mathrm{L})$ antibody (Invitrogen Molecular Probes, A11029). Images were acquired on the TyphoonTM Scanner (Amersham Bioscience), and quantified using ImageQuant TL v2005 Software.

28. Wallace, E. M. et al. Potent and selective mitogen-activated protein kinase kinase (MEK) 1,2 inhibitors. 1. 4-(4-bromo-2-fluorophenylamino)-1- methylpyridin2(1H)-ones. J. Med. Chem. 49, 441-444 (2006).

29. Cheng, Y. \& Prusoff, W. H. Relationship between the inhibition constant $\left(K_{l}\right)$ and the concentration of inhibitor which causes 50 per cent inhibition $\left(I_{50}\right)$ of an enzymatic reaction. Biochem. Pharmacol. 22, 3099-3108 (1973).

30. Lukyanov, K. A. et al. Natural animal coloration can be determined by a nonfluorescent green fluorescent protein homolog. J. Biol. Chem. 275, 25879-25882 (2000).

31. Matz, M. V. et al. Fluorescent proteins from nonbioluminescent Anthozoa species. Nature Biotechnol. 17, 969-973 (1999).

32. Stokoe, D. \& McCormick, F. Activation of c-Raf-1 by Ras and Src through different mechanisms: activation in vivo and in vitro. EMBO J. 16, 2384-2396 (1997).

33. Collaborative Computational Project, Number 4. The CCP4 suite: programs for protein crystallography. Acta Crystallogr. D 50, 760-763 (1994). 